\title{
Place de la salive et des cheveux dans le dépistage d'un usage de stupéfiants en milieu professionnel
}

\section{Place of oral fluid and hair for workplace drug testing}

Nele SAMYN*(1), Vincent ARESCHKA ${ }^{(1)}$, Pascal KINTZ(2)

(1) Institut National de Criminalistique et de Criminologie (I.N.C.C.), Chaussée de Vilvorde 100, 1120 BRUXELLES, Belgique

(2) Institut de Médecine Légale, 11, rue Humann - 67000 STRASBOURG

* Auteur à qui adresser la correspondance : Nele SAMYN, Institut National de Criminalistique et de Criminologie (I.N.C.C.), Chaussée de Vilvorde - 100, 1120 BRUXELLES, Belgique Tél : +32 22400500 - Fax : +32 22416105 - e-mail : nele.samyn@ just.fgov.be ou nele.samyn@kbcmail.net

(Reçu le 1'er février 2002 ; accepté le 15 février 2002)

\section{RÉSUMÉ}

La procédure standard d'une recherche de stupéfiants en milieu professionnel consiste en un criblage d'un échantillon d'urine par une technique immunochimique suivie d'une confirmation par chromatographie en phase gazeuse couplée à la spectrométrie de masse (CG/SM). Cette technique d'analyse en deux étapes est consensuelle, et apparaît applicable à d'autres fluides biologiques. En effet, ces dernières années, les progrès remarquables des techniques analytiques en terme de sensibilité ont permis l'identification de stupéfiants à partir de matrices non-conventionelles telles la salive et les cheveux. Le principal avantage de ces matrices est un prélèvement non invasif qui peut se faire sous surveillance étroite, diminuant ainsi les risques de modifications ou de substitutions des échantillons. La présence d'un stupéfiant dans la salive peut être consécutif à l'excrétion du produit à partir du

\section{SUMMARY}

The standard in workplace drug testing has been the immunoassay screen followed by the gas chromatography/mass spectrometry (GC/MS) confirmation conducted on a urine sample. This two-step drug testing technology is generally accepted and is embedded into a comprehensive system of workplace drug testing also allowing the use of other bodyfluids. In recent years, remarkable advances in sensitive analytical techniques have enabled the analysis of drugs in unconventional biological specimens such as saliva and hair. The main advantage of these matrices over conventional media is obviously the non-invasive collection protocol, which can be achieved under close supervision to prevent adulteration or substitution of the samples.

Drugs appear in oral fluid via multiple pathways e.g. excretion from blood and contamination of the oral cavity during 
sang ou à une contamination de la cavité buccale lors de la consommation par voie orale, sniffée ou fumée. Un protocole de recueil salivaire bien défini et reproductible est important pour permettre l'interprétation des concentrations salivaires. Une approche immunochimique spécifique et sensible a été développée pour le criblage au niveau du laboratoire. Compte tenu d'un volume d'échantillon relativement faible ( 1 à $2 \mathrm{ml}$ ) et des faibles concentrations retrouvées dans la salive, il est nécessaire de faire appel à des techniques chromatographiques très sensibles telles la CG/SM/SM, la CLHP/SM/SM ou la CG/SM/ICN pour la confirmation. Un test salivaire positif peut raisonnablement être associé à une consommation récente, globalement jusqu'à 12 à 24 heures avant le dépistage. Des études récentes ont démontré que la recherche de $\Delta$-9-tétrahydrocannabinol dans la salive peut révéler une consommation récente d'une dose unique de marijuana alors même que l'analyse d'un échantillon d'urine pourrait s'avérer négative jusque 4 à 6 heures après avoir fumé un joint. En ce qui concerne l'analyse des stupéfiants dans les cheveux, les résultats sont significatifs des semaines, voire des mois, après consommation, ce qui pourrait être très utile, par exemple, dans le cas de dépistage de futurs collaborateurs au sein $>$ d'une entreprise. De nombreuses substances psycho-actives ont été identifiées dans les cheveux. Comme pour la salive, la recherche des stupéfiants dans les cheveux doit cibler la substance mère, que ce soit pour le dépistage ou pour la confirmation. Certains aspects de l'interprétation des analyses sont encore en discussion : difficulté à détecter un usage récent, influence de la couleur des cheveux, de l'origine ethnique et du sexe, et distinction entre utilisation active et exposition passive. Des programmes d'assurance qualité pour les cheveux, la salive et la sueur commencent à être initiés aux Etats-Unis et en Europe (Society of Hair Testing) avec des recommandations de sociétés savantes, comme par exemple, l'emploi de seuils de positivité pour le dépistage et la confirmation.

\section{MOTS-CLÉS}

Salive, cheveux, stupéfiants, dépistage, milieu professionnel.

\section{Introduction}

Compte tenu du grand nombre de consommateurs de drogues dans la population active aux Etats-Unis et les risques que représentent ces derniers tant pour euxmêmes que pour autrui, leur dépistage en milieu professionnel est devenu chose courante dans ce pays. En Europe, ce dépistage est peu fréquent alors que les diverses tendances en matière de consommation de drogue et de trafic international se ressentent nettement dans nos sociétés. De plus, la législation en la matière dans les différents états européens est peu homogène et il n'existe généralement pas de consensus concernant les procédures à adopter (1). En effet, le choix de la matrice pour effectuer une recherche de stupéfiants est influencé par une variété de facteurs tels que la facilité de prélèvement de l'échantillon, des considérations purement analytiques ainsi que par le niveau d'exploitation des résultats que l'on recherche. Longtemps oral, intranasal and smoked administration. A reproducible, well-defined collection protocol is important to simplify the interpretation of quantitative results. Specific and sensitive micro-plate Enzyme immunoassays are developed for screening of oral fluid on a laboratory basis. For confirmation analysis, due to the lower sample volume available and the lower concentrations detected in saliva, in comparison to urine, very sensitive chromatographic techniques like GC$M S-M S, L C-M S-M S$ and GC-MS-NCI should be used. A positive result from an oral fluid test can be interpreted as being attributable to recent drug use, generally within 12-24 $h$. Recent studies have shown that oral fluid testing for $\Delta-9$ tetrahydrocannabinol can pick up recent use of a single dose of marihuana whereas the corresponding urine sample might be negative for 4-6 hrs after smoking. In the case of hair, the window of drug detection is dramatically extended to weeks or months which could be useful e.g. in pre-employment testing. A wide variety of drugs has been detected in hair. As for oral fluid, drug testing in hair should focus on the parent drug; similar analytical techniques should be used for screening and confirmation. Some issues that remain partially unresolved are : the difficulty to detect recent drug use, the influence of color, ethnic origin and sex on the concentrations of some analytes, and the distinction between active use and passive exposure. A performance testing program for laboratories was initiated in the US, recently followed by the Society of Hair Testing with draft guidelines for oral fluid, sweat and hair, proposing for example meaningful cut-off values for screening and confirmation.

\section{KEY-WORDS}

Saliva, oral fluid, hair, drugs of abuse, screening, workplace drug testing.

limité à l'analyse des urines, l'intérêt en matière de dépistage de drogues en milieu professionnel s'est récemment porté sur d'autres matrices offrant des avantages distincts, ce qui a permis l'apparition de campagnes de dépistages plus efficaces (2). Le prélèvement des échantillons de salive (fréquemment dénommée oral fluid aux États-Unis) et de cheveux est plus aisé et surtout moins invasif que pour l'urine et le sang ; la facilité de contrôle du prélèvement permet d'éviter toute suspicion en matière de falsification éventuelle de ces échantillons. Les informations issues de l'analyse de ces matrices alternatives peuvent être très utiles pour une interprétation plus fine du profil de consommation de drogue d'un individu : l'histoire de la consommation de drogues peut être établie par l'analyse des cheveux et l'état d'intoxication peut être mieux estimé du fait d'une meilleure corrélation (par rapport à l'urine) entre les concentrations sanguines et salivaires. 
Les techniques traditionnelles peuvent être utilisées pour la détection des stupéfiants dans les matrices alternatives ; cependant et par rapport aux urines, certaines limitations sont imposées par le fait que le volume ou la quantité de l'échantillon disponible est généralement faible, que les substances cibles sont différentes et que le traitement de l'échantillon peut s'avérer plus complexe. Bien que l'évolution remarquable en matière de sensibilité des techniques analytiques séparatives permette actuellement la détection spécifique de nombreuses substances psychotropes dans la salive et les cheveux, seuls des progrès modestes ont été observés au niveau commercial dans le développement d'outils de prélèvement, de tests de criblage sur site et de laboratoire, de matériel spécifique en matière de contrôle de qualité, en matière de programmes d'assurance qualité et en matière de recommandations de sociétés savantes.

\section{Développement de direc- tives pour le dépistage des stupéfiants dans la salive et les cheveux en milieu pro- fessionnel}

Depuis le milieu des années 80 , des directives précises ont été publiées pour l'urine en matière de prélèvement des échantillons, de traçabilité, d'analyse (criblage et confirmation), de certification des laboratoires, d'interprétation des résultats ainsi qu'en matière de contreexpertise $(3,4)$. Dans le même ordre logique, les pays qui commencent à utiliser les matrices alternatives devraient aussi développer un ensemble des directives similaires à celles existantes pour les urines. A l'heure actuelle, ces procédures s'élaborent lentement dans la plupart des pays concernés. Parfois, il est suggéré d'employer les tests d'urine et de salive dans le cas de dépistage de drogue pour de futurs collaborateurs au sein d'une entreprise, suite à un accident de travail, en cas de doutes raisonnables, comme tests de contrôle aléatoires, éventuellement suite à une absence prolongée. L'emploi des cheveux serait recommandé pour le dépistage de futurs collaborateurs, suite à une absence prolongée et éventuellement comme tests de contrôle aléatoire. La force d'un nouveau programme de dépistage réside en sa capacité à intégrer les adaptations consécutives aux progrès technologiques effectués et validés en la matière. Les procédures de prélèvement, de stockage et de transport de ces nouveaux types d'échantillons doivent tenir compte des exigences et des restrictions imposées par chaque matrice. Les tests de criblages et les analyses de confirmation doivent être développés ou peuvent être dérivés de procédures existantes permettant ainsi le dépistage spécifique des analytes cibles. Des valeurs seuil scientifiquement et légalement défendables doivent aussi être établies. Certaines recommandations ont été proposées dans ce domaine par le programme international de contrôle des drogues des Nations Unies (UNDCP) et par une division du SAMHSA (Substance Abuse and Mental Health Service Administration) aux États-Unis $(5,6)$. Les laboratoires utilisant ces matrices alternatives doivent êtres capables d'identifier les analytes cibles et d'en mesurer précisément les concentrations. Le SAMHSA a organisé un premier exercice de trois cycles (chaque fois vingt échantillons) pour le criblage et la confirmation des stupéfiants dans la salive, la sueur et les cheveux. Elle s'est référée aux valeurs seuil proposées par les différents groupes de travail (6). Proposés en 1992 par le National Institute of Standards and Technologies (États-Unis), plusieurs exercices visant le contrôle de qualité des analyses de cheveux ont été récemment fédérés par une société savante, la Society of Hair Testing (SOHT). Cette dernière essaie d'arriver à un consensus pour le prélèvement des échantillons, pour l'interprétation des résultats ainsi que pour la recherche de valeurs seuil et de substances précises permettant de faire la distinction entre une consommation de drogue et une exposition passive (7). Des exercices nationaux, sous l'égide de la SFTA, ont porté sur des échantillons réels (8). Au niveau purement législatif il est rare qu'une autorisation spécifique ou même la mention de ces matrices alternatives soit présente. De rares pays disposent cependant de lois interdisant spécifiquement l'usage des cheveux et de la salive comme outil de dépistage de consommation de drogue (9). En France, la loi Buffet autorise les cheveux dans le cadre du suivi de sportifs dopés.

\section{Pharmacocinétique drogues dans la salive \\ des}

Le tableau I présente les caractéristiques les plus importantes d'une analyse de salive par rapport aux traditionnelles analyses d'urines. Il est recommandé de prélever un deuxième échantillon dans l'éventualité d'une contre-expertise. Le volume exact collecté est plus difficile à mesurer pour la salive que pour les urines, ce qui est inhérent à la plupart des matrices alternatives. Généralement, un résultat positif issu d'un test de salive peut être interprété avec un degré de certitude élevé comme résultant d'une consommation récente de produit, dans les 24 heures précédant le prélèvement, et non comme résultant d'une consommation 
ayant eu lieu des jours, voire des semaines avant le test (problème associé au dépistage du cannabis dans les urines). Les stupéfiants peuvent apparaître dans la salive par de multiples voies, dont essentiellement la diffusion passive du sang vers la salive et la contamination directe de la cavité buccale suite à une consommation orale, intranasale ou fumée. C'est le caractère non invasif associé au recueil de la salive qui est à l'origine depuis plusieurs années du grand intérêt porté à cette matrice comme outil analytique en toxicologie ; un nombre substantiel de revues de la littérature a été publié sur ce sujet, tant pour les substances licites qu'illicites (10-13). Le présent article se concentrera sur certaines études expérimentales récentes où des administrations contrôlées ont été pratiquées sur des volontaires, fournissant des données fiables en matière de cinétique d'apparition des drogues dans la salive en parallèle avec l'urine ou le sang.

\section{Cannabinoïdes}

Suite à une contamination orale, des résidus de $\Delta 9$ tétrahydrocannabinol (THC) peuvent être piégés dans les tissus de la sphère buccale. Le transfert du THC du sang vers la salive est minimal. Une étude menée sur une population de conducteurs ciblée a montré des concentrations variables de THC en fonction de la méthode de prélèvement utilisée (14), mais jamais la présence des métabolites (11OH-THC ou THC$\mathrm{COOH}$ ) pendant les 7 jours suivant la dernière consommation. Dans une étude récemment publiée (15), 18 volontaires, consommateurs chroniques ou occasionnels de marijuana, ont fumé une cigarette contenant 20 à $25 \mathrm{mg}$ de THC. L'étude a permis le recueil de la salive et des urines des sujets consommant de la marijuana dans un contexte social réel. Les échantillons de salive ont été collectés avec un outil de collection spécial (Intercept ${ }^{\circledR}$, Orasure Technologies, USA) composé d'un collecteur et d'une solution de conservation qui dilue la salive d'un facteur 3. Les échantillons de salive ont révélé, par analyse CG/SM (seuil de $0,5 \mu \mathrm{g} / \mathrm{l}$ ), du THC pendant $13 \pm 3$ heures après la consommation de marijuana. Les échantillons d'urine ont été positifs par $\mathrm{CG} / \mathrm{SM}$ durant une période moyenne de $33 \pm 10$ heures en utilisant la valeur seuil SAMHSA de $15 \mu \mathrm{g} / \mathrm{l}$ pour le métabolite : l'acide 11-nor- $\Delta$-9-tétrahydrocannabinol-

Tableau I : Caractéristiques générales des différentes matrices en matière de dépistage de consommation de drogue.

\begin{tabular}{|c|c|c|}
\hline Matrice & Avantages & Désavantages \\
\hline Urine & $\begin{array}{l}\text { - concentration élevée des drogues et de leur(s) } \\
\text { métabolite(s) spécifique(s) } \\
\text { - techniques de criblages commerciales fiables } \\
\text { - critères analytiques uniformes établis (valeurs seuil p. ex.) } \\
\text { - tests de contrôle de performance largement pratiqués } \\
\text { - recommandations pour l'interprétation des résultats } \\
\text { et leur acceptation au tribunal }\end{array}$ & $\begin{array}{l}\text { - protocole de collection invasif } \\
\text { - possibilité d'adultération de l'échantillon in-vitro } \\
\text { et in-vivo, substitution } \\
\text { - période de détection de } 2 \text { à } 3 \text { jours } \\
\text { - pas de relation dose/concentration } \\
\text { - pas de relation concentration/modification de la vigilance }\end{array}$ \\
\hline Salive & $\begin{array}{l}\text { - facilité de prélèvement } \\
\text { - suivi du recueil aisé } \\
\text { - détection de la substance mère et de ses métabolites } \\
\text { - utile pour la détection d'une consommation récente } \\
\text { - résultats pouvant être associés avec une consommation } \\
\text { datant de } 12 \text { à } 24 \text { heures }\end{array}$ & $\begin{array}{l}\text { - volume collecté faible } \\
\text { - volume collecté dépendant de l'outil de collection } \\
\text { - les concentrations observées sont influencées par la voie } \\
\text { d'administration (contamination orale) et par le protocole } \\
\text { de prélèvement (pH salivaire) } \\
\text { - la fenêtre de détection peut-être plus courte } \\
\text { - tests de contrôle de performance en cours de développement }\end{array}$ \\
\hline Cheveux & $\begin{array}{l}\text { - suivi du recueil aisé } \\
\text { - facilité d'obtention, de stockage et de transport } \\
\text { des prélèvements } \\
\text { - possibilité de tracer une consommation de drogue } \\
\text { sur une longue période } \\
\text { - détection de la substance mère et de ses métabolites } \\
\text { - possibilité d'obtenir un second échantillon à partir } \\
\text { du donneur original }\end{array}$ & $\begin{array}{l}\text { - difficulté à détecter un usage récent } \\
\text { - incorporation fonction de la pigmentation des cheveux } \\
\text { - contamination externe des cheveux possible pour certaines } \\
\text { classes de drogues } \\
\text { - adultération possible de l'échantillon avant prélèvement } \\
\text { - relation dose/concentration (?) } \\
\text { - tests de contrôle de performance en cours de développement }\end{array}$ \\
\hline
\end{tabular}


carboxylique (THC-COOH). Contrairement à la salive, l'analyse d'urine se révèle généralement négative immédiatement après la consommation de marijuana et n'est que positive en moyenne après $4 \pm 1$ heures. La concentration salivaire de THC diminue rapidement durant les premières heures après ingestion orale ou fumée. Des variations individuelles substantielles ont été constatées dans les profils d'élimination. L'analyse de prélèvements effectués simultanément du côté gauche et droit de la cavité buccale a révélé une excellente cohérence. Malgré le faible apport sanguin de THC dans la salive, l'évolution de la concentration de THC en fonction du temps dans la salive et le sang est remarquablement similaire si l'on tient compte des concentrations plasmatiques reportées par Huestis et al. (16). La durée de détection moyen du THC dans le plasma (valeur seuil de $0,5 \mu \mathrm{g} / \mathrm{l}$ ) est de 7,2 et 12,5 heures suite à la consommation d'une cigarette de marijuana contenant respectivement une dose de 15,8 et $33,8 \mathrm{mg}$ de THC.

En conclusion, la réussite du dépistage d'une consommation de marijuana utilisant la salive est certainement lié à l'efficacité de l'outil de prélèvement, qui doit assurer un recueil reproductible. L'analyse de la salive se montre plus efficace pour la détection de cas très récents de consommation de marijuana par rapport à l'analyse des urines et généralement, une fréquence de détection plus élevée est observée par analyse de la salive durant les huit premières heures. Cette caractéristique unique de la salive est importante si l'on tient compte de l'activité limitée dans le temps de la marijuana sur la vigilance. Finalement, la corrélation existante entre le plasma et la salive suggère que la salive est un meilleur indicateur que l'urine pour démontrer une consommation active de stupéfiant.

\section{Amphétamines}

Dans deux études contrôlées récentes $(17,18)$, des volontaires se sont vus administrer une dose unique (75 - $100 \mathrm{mg}$ ) de 3,4-méthylènedioxy-N-méthylamphétamine (MDMA, «ecstasy»). Une bonne corrélation a été observée pour les profils d'évolution moyen de la concentration sanguine et salivaire de MDMA. Cependant, une variabilité plus grande a été constatée pour la salive entre les différents participants $(17,18)$, avec des concentrations maximales de MDMA variant de 1728 à $6510 \mu \mathrm{g} / \mathrm{l}$ une heure et demie après administration, et chutant à une valeur moyenne de $126 \mu \mathrm{g} / \mathrm{l}$ après 24 heures (17). Les concentrations de MDMA observées 5 heures après administration ont une valeur moyenne de $526 \pm 372 \mu \mathrm{g} / \mathrm{l}$ dans la salive et de $13 \pm 8$ $\mathrm{mg} / \mathrm{g}$ de créatinine dans l'urine (18). Les échantillons de salive ont été collectés en faisant cracher les partici- pants sans aucune forme de stimulation ; le $\mathrm{pH}$ salivaire a été déterminé dans une étude (17). Le $\mathrm{pH}$ salivaire semble être affecté par une consommation de MDMA : le $\mathrm{pH}$ chute de 7,4 à une valeur moyenne de 6,8 une heure et demie après administration pour retourner à une valeur de 7,3 après 6 heures. En conséquence, les concentrations salivaires observées après une heure et demie sont en moyenne 10 fois supérieures aux concentrations observées dans le plasma ; elles se stabilisent à une valeur plus faible (4-7) après 5 à 24 heures $(17,18)$.

En conclusion, la salive présente une plus grande fenêtre de temps de détection d'une consommation de MDMA que le plasma. Le caractère non-invasif de l'échantillonnage, les concentrations relativement élevées de MDMA dans la salive en phase de post-absorption et la forte influence du $\mathrm{pH}$ urinaire sur l'excrétion urinaire des amphétamines, rendent le prélèvement de salive préférable au prélèvement d'urine.

\section{Cocaïne}

L'équipe de Cone (19) a publié l'étude la plus complète concernant l'excrétion de la cocaïne dans la salive. Différentes voies d'administration font apparaitre rapidement la cocaïne dans la salive. La contamination de la cavité buccale suite à une consommation sous forme fumée ou sniffée est variable mais significative durant les premières heures consécutives à l'administration d'une dose unique ; il en résulte un rapport salive / plasma (S/P) élevé. La benzoylecgonine (BE) et l'ester méthylique de l'ecgonine apparaissent dans la salive généralement entre 0,08 heure et 1 heure après administration. Typiquement, la concentration de cocaïne est supérieure à celle des métabolites durant les deux premières heures ; elle décline ensuite rapidement à des concentrations parfois inférieures à celles des métabolites dans les 4 à 6 heures. Il a été rapporté pour la $\mathrm{BE}$ un rapport $\mathrm{S} / \mathrm{P}$ moyen de $0,53 \pm 0,64$ chez des conducteurs sous influence (14). Moolchan et al. (20) ont étudié la pharmacocinétique de la cocaïne et de la benzoylecgonine dans la salive et le plasma de consommateurs chroniques de cocaïne en période de sevrage. Pour la cocaïne, des temps de demi-vie de 3,8 heures ont été mesurés dans le plasma et de 7,9 heures dans la salive. Ces valeurs sont respectivement de 6,6 heures et de 9,2 heures pour la benzoylecgonine. Cette étude suggère une élimination prolongée de la cocaïne chez les consommateurs réguliers par rapport aux consommateurs occasionnels bien que le temps de demi-vie du métabolite principal de la cocaïne, la benzoylegonine, reste similaire. Une étude supervisée par les autorités judiciaires a montré que la salive présente une sensibilité de $100 \%$ et une spécificité de $99 \%$ pour la détec- 
tion d'une consommation de cocaïne par rapport aux analyses d'urines utilisées comme référence (21).

En conclusion, sachant que la concentration de cocaïne dans la salive est étroitement dépendante du $\mathrm{pH}$ et d'une possible contamination de la cavité buccale, l'emploi de la benzoylecgonine comme molécule cible pour le criblage de la salive en vue de la détection d'une consommation relativement récente de cocaïne s'avère plus pertinent et offre de surcroît une fenêtre de détection plus large.

\section{Opiacés}

Tout comme pour la cocaïne, l'héroïne et ses métabolites -à savoir la 6-monoacétylmorphine (6-MAM), la morphine et la codéine- sont détectés dans la salive après administration intraveineuse et fumée. Les concentrations mesurées sont fonction de la voie d'administration ainsi que du $\mathrm{pH}$ salivaire et semblent évoluer parallèlement aux concentrations sanguines $(22,23)$. Une corrélation supérieure à $90 \%$ entre la salive et les urines a été observée par Niedbala et al. (24) en appliquant les valeurs seuil du SAMHSA pour les urines et une valeur de positivité de $10 \mu \mathrm{g} / \mathrm{l}$ pour un ou plusieurs opiacés présents dans la salive. La salive a été recueillie avec le collecteur Intercept ${ }^{\circledR}$; il dilue la salive d'un facteur trois et assure une stabilité de 90 jours pour la morphine et d'une semaine pour la 6-MAM. Cette procédure permet le transfert aisé des échantillons au laboratoire.

L'influence des protocoles de recueil sur la concentration salivaire de codéine a été évaluée après administration contrôlée (25). Les concentrations sont plus élevées lorsque l'on ne stimule pas la production de salive, cette dernière étant donc recueillie crachée. Les concentrations sont plus faibles lorsque la production de salive est stimulée mécaniquement ou chimiquement ou encore quand la salive est collectée avec des collecteurs tel que la Salivette ${ }^{\circledR}$ (Sarstedt, Allemagne) ou le doigt de receuil Accusorb ${ }^{\circledR}$ de Avitar Technologies (USA). Tout comme pour le THC, il a été démontré l'importance du rendement d'extraction des analytes cibles de ces outils de collection.

En conclusion, la détection des opiacés dans les échantillons de salive fournit des résultats comparables aux urines. Les concentrations salivaires de 6-acétylmorphine et de morphine sont presque égales et facilement confirmés par les méthodes conventionnelles de CG/SM. Les échantillons de salive analysés après ingestion de graines de pavot se différencient de ceux des utilisateurs d'héroïne par l'absence de 6-monoacétylmorphine.

\section{Pharmacocinétique des drogues dans les cheveux}

Depuis 1979, les cheveux ont été employés pour identifier des consommations chroniques de xénobiotiques. La littérature rapporte plus de 400 publications concernant l'analyse des cheveux dans des domaines aussi variés que la toxicologie médico-légale (26), la toxicologie clinique (27), la médecine du travail (28) et le dopage (29). Le tableau II présente une comparaison des caractéristiques les plus importantes de l'analyse des cheveux. Cette matrice offre certains avantages pour les employeurs de même que pour les employés, compte tenu du caractère non-traumatique des prélèvements, du statut relativement inerte de la matrice ainsi que de la possibilité de procéder à un autre moment à un second prélèvement recouvrant la même période de temps que le premier. La possibilité unique qu'offrent les cheveux de retracer une consommation de drogue les rendent nettement préférables aux urines pour le dépistage de futurs collaborateurs au sein de l'entreprise ; la période de temps couverte par le dépistage dépend cependant de la longueur de l'échantillon capillaire prélevé (chaque $\mathrm{cm}$ représente environ 1 mois de pousse). Pour les urines, une période d'abstinence de quelques jours est généralement suffisante pour influencer fondamentalement les résultats des tests. L'analyse des cheveux permet aussi aux employés de démontrer leur bonne foi en prouvant par exemple qu'ils ont surmonté leurs problèmes de toxicomanie. En pratique, les fenêtres de détection offertes par les urines et les cheveux sont complémentaires. Bien qu'il existe un consensus raisonnable concernant la validité des analyses qualitatives des cheveux, l'interprétation des résultats peut être délicate car il existe encore des questions non résolues telles que l'influence de la contamination externe (30), des traitements cosmétiques (31) et de possibles facteurs raciaux dont pourraient dépendre l'incorporation des drogues dans les cheveux (32).

Tableau II : Seuils proposés pour l'interprétation des résultats des analyses de cheveux.

\begin{tabular}{|l|c|}
\hline analyte & seuil de positivité \\
\hline 6-MAM & $0.5 \mathrm{ng} / \mathrm{mg}$ \\
Cocaïne & $0.5 \mathrm{ng} / \mathrm{mg}$ \\
THC-COOH & $1 \mathrm{pg} / \mathrm{mg}$ \\
amphétamines & $0.5 \mathrm{ng} / \mathrm{mg}$ \\
\hline
\end{tabular}




\section{Incorporation des drogues dans les cheveux}

Le délai d'apparition d'une substance dans les cheveux est caractérisé par une variabilité considérable entre individus ; il varie de un jour à une semaine. Les voies d'incorporation incluent la diffusion à partir du sang, de la sueur, du sébum et la contamination externe. L'incorporation d'un xénobiotique dans le cheveu est principalement régie par son caractère lipophile, son caractère basique ainsi que par l'affinité que présente cette substance pour la mélanine. La mélanine et certaines protéines naturellement présentes dans le cheveu peuvent servir de site de fixation. La pigmentation du cheveu ou son contenu en mélanine, et par conséquent la couleur et l'origine ethnique des cheveux, semble être un des paramètres les plus importants régissant l'incorporation. A dose administrée équivalente, il a été démontré que les cheveux noirs, donc contenant beaucoup de mélanine, présentent des concentrations plus élevées que les cheveux blonds (32). Par contre, il n'existe pas de consensus sur le fait que les variations de concentrations observées dans les cheveux entre individus soient substantiellement différentes des variations de concentrations urinaires pondérées par le contenu aqueux (33). Certains traitements cosmétiques peuvent modifier la concentration de stupéfiants présente dans les cheveux. Les décolorations ou colorations ainsi que les «permanentes» diminuent de façon très importante $(40$ à $80 \%)$ la concentration des drogues présentes dans les cheveux (31), rendant de la sorte possible une méthode d'adultération avant le prélèvement d'un échantillon capillaire. Le simple fait de se laver les cheveux avec certains produits peut faire chuter nettement la concentration de certaines drogues ; on observe ainsi, en un seul lavage, une diminution de $36 \%$ du THC contenu dans les cheveux avec le shampoing «Ultra Clean» par exemple (34).

Les cheveux en croissance (environ $85 \%$ de la quantité totale) incorporent les substances présentes dans le sang et la sueur et peuvent ainsi représenter le calendrier rétrospectif de la consommation chronique d'un xénobiotique. En effet, les cheveux poussent d'environ 1 centimètre par mois et leur analyse centimètre par centimètre, de la racine (consommation la plus récente) vers la pointe des cheveux (consommation la plus ancienne dans le temps) permet de suivre l'évolution de la consommation mois après mois. Néanmoins, il faut garder en mémoire que la croissance des cheveux peut varier d'une personne à l'autre, qu'elle n'est pas continue et que des phénomènes de migration à l'intérieur du cheveu peuvent affecter les concentrations.

\section{Importance de la contamination externe}

Les substances fumées comme la nicotine, le cannabis, le «crack» ou même l'hérö̈ne peuvent se déposer sur toute la longueur du cheveu. Si l'incorporation via le sang se fait dans la région médullaire de la matrice kératinisée, dans le cas d'une contamination passive, elle pourrait intervenir plus superficiellement au niveau cortical. Les substances déposées sur les cheveux par voie passive seraient alors moins bien liées à la matrice, ce qui a conduit les toxicologues à développer des méthodes de décontamination des échantillons. Elles consistent en des lavages, soit par une solution aqueuse, soit par un solvant organique, soit par les deux successivement pendant différents temps d'incubation et à différentes températures. Des cinétiques de lavage et l'analyse des solutions de décontamination ont révélé que les contaminants étaient très vite éliminés (après deux lavages) et qu'ensuite, d'autres lavages n'avaient plus aucun effet. La diversité des protocoles de décontamination ont conduit les toxicologues à rechercher les produits de dégradation métabolique de la substance mère, ne pouvant provenir ni de la contamination environnementale, ni de l'hydrolyse de l'échantillon lors de l'analyse. La détection des marqueurs spécifiques (cocaethylène, norcocaine) n'est pas aisée car leurs concentrations sont généralement très faibles. Certains laboratoires utilisent des ratio de métabolites pour éviter des faux positifs. Finalement, la mise en place de seuils de positivité a permis d'interpréter de façon plus aisée les résultats obtenus.

\section{Interprétation des résultats quantitatifs pour différentes classes de drogues}

Kintz a proposé certaines valeurs seuil pour l'interprétation des résultats des analyses quantitatives dans les cheveux (tableau II) (35). En fait, il apparaît que chaque laboratoire, sur la base de ses propres études de population, devrait déterminer ses propres valeurs seuil en fonction de son pays d'origine, du but des analyses, des substances cibles recherchées et de la méthode analytique employée. L'interprétation liée à l'application d'une valeur seuil pour la cocaïne peut être affinée dans certains cas par la détection de la norcocaïne ou du cocaéthylène, formés au niveau hépatique lors de la consommation concomitante de cocaine et d'alcool éthylique. Cone et al. (36) ont proposé un rapport $\mathrm{BE} /$ cocaine $>0,05$ comme étant en faveur d'une consommation active de cocaïne. Un rapport de concentration 6-MAM/morphine de minimum 1,3 est recommandé par la SOHT comme indicatif d'une consommation active d'hérö̈ne. Pépin et Gaillard (37) ont corrélé les plages de concentrations de 6-MAM et de cocaïne dans les cheveux en fonction du niveau de consommation (tableau III). Bien que le THC-COOH soit présent en moindre concentration (et cela d'un facteur 1000 à 10000) que le THC, le cannabinol et le cannabidiol, son identification dans le cheveu constitue une preuve définitive d'une consommation de marijua- 
na car cette substance est absente de la fumée de marijuana.

L'étude statistique des données issues des programmes de dépistage dans le milieu professionnel aux USA pour l'année 2000 révèle une concentration moyenne de métamphétamine de $1,83 \mathrm{ng} / \mathrm{mg}$ de cheveux. Une valeur seuil de $0,5 \mathrm{ng} / \mathrm{mg}$ de cheveux est employée pour cette substance. La valeur seuil de l'amphétamine est abaissée à $0,05 \mathrm{ng} / \mathrm{mg}$ de cheveux afin de pouvoir permettre l'interprétation des résultats en fonction du rapport de concentration métabolite/substance mère dans le cas d'une éventuelle consommation de métamphétamine. Pour la MDMA, une valeur seuil de 0,1 à $0,5 \mathrm{ng} / \mathrm{mg}$ de cheveux a été suggérée et le seul métabolite présent est la MDA, dont la présence n'est pas suffisamment spécifique étant donné qu'il existe des pilules contenant du MDA.

Tableau III : Plages de concentrations observées dans les cheveux en fonction de la fréquence de consommation de cocaïne et d'héroüne, dans le cas de consommations rapportées.

\begin{tabular}{|l|c|c|c|c|}
\hline \multirow{2}{*}{ analyte } & valeur seuil & \multicolumn{3}{|c|}{ Fréquence de consommation } \\
\cline { 3 - 5 } & & Faible & Intermédiaire & Élevée \\
\hline 6-MAM & $0.5 \mathrm{ng} / \mathrm{mg}$ & $<2 \mathrm{ng} / \mathrm{mg}$ & $2-10 \mathrm{ng} / \mathrm{mg}$ & $>10 \mathrm{ng} / \mathrm{mg}$ \\
Cocaïne & $1 \mathrm{ng} / \mathrm{mg}$ & $<4 \mathrm{ng} / \mathrm{mg}$ & $4-20 \mathrm{ng} / \mathrm{mg}$ & $>20 \mathrm{ng} / \mathrm{mg}$ \\
\hline
\end{tabular}

\section{Limites de détection comparativement aux urines}

Une étude (33) a comparé l'efficacité des analyses de dépistage effectuées sur les cheveux et les urines pour la cocaïne et la marijuana. Le dépistage de la cocaïne est le plus performant des tests capillaires compte tenu des grandes concentrations de cocaïne présentes dans les cheveux. Le dépistage de la marijuana dans les cheveux est nettement plus difficile car les concentrations de THC-COOH dans les cheveux sont de l'ordre de $1 \mathrm{pg} / \mathrm{mg}$, ce comparativement à la fenêtre de détection étendue et aux concentrations élevées qu'offrent les urines. Les résultats ont été interprétés selon que les échantillons aient été collectés : a/ en l'absence totale de possibilité d'adultération (au moment de l'arrestation par exemple) b/ en l'absence de possibilité d'adultération aisé (en cas de test aléatoire non communiqué) c/ en présence de réelles possibilités d'adultération (test lié aux recrutements). Les tests de dépistage de la marijuana dans les cheveux se sont montrés aussi performants que les tests d'urines même quand les urines ont été collectées comme décrit en a/, à savoir en l'absence totale de possibilité d'adultération. Les tests capillaires se sont montrés de 35 à $600 \%$ plus efficaces que les tests d'urine lorsque les échantillons ont été collectés comme décrit en b/ et en c/. Concernant la cocaïne, la détection liée aux tests capillaires est respectivement $92 \%, 272 \%$ et $1580 \%$ plus efficace suivant que les échantillons aient été prélevés dans les conditions a, $\mathrm{b}$ et c. Pour les analyses d'urines, les consommateurs occasionnels de cocaïne, par comparaison avec les consommateurs chroniques, présentent une probabilité nettement moins élevée d'être testés positifs dans la fenêtre de détection de trois jours qu'offre cette matrice en cas de contrôle non annoncé. La comparaison des résultats issus des tests d'urines et de cheveux en fonction du type de consommation, montre que les tests de cheveux sont $670 \%$ plus efficaces pour le dépistage des consommateurs occasionnels. Il semble exister une corrélation entre la dose ingérée et la concentration mesurée dans les cheveux pour la plupart des cocaïnomanes ; une distinction entre consommateurs occasionnels, moyens et chronique est donc possible.

\section{Techniques de criblages et analyses de confirmation des drogues dans la salive et les cheveux}

Bien que les analyses dans le domaine des matrices alternatives aient été effectuées initialement au moyen de techniques radioimmunologiques, de nombreuses nouvelles variantes commerciales non-isotopiques sont actuellement disponibles sur le marché pour le criblage des drogues et des métabolites de drogues. Des techniques telles que l'ELISA (Enzyme linked immunosorbent assay) ont été adaptées pour la détection des analytes dans les extraits/les produits de digestion des cheveux ainsi que dans la salive. La plupart de ces tests ont une sensibilité raisonnable à élevée pour la molécule mère. Spiehler a passé en revue les méthodes immunochimiques existantes pour le criblage des cheveux (38) et a démontré que la matrice en solution provoque des interférences importantes. Les tests immunochimiques utilisant des anticorps immobilisés sur les parois en plastique du test, de même que les supports qui permettent d'éliminer la matrice par lavage après la réaction de compétition permettent l'obtention de bons résultats. Des études intéressantes portant sur la salive ont révélé une spécificité et une sensibilité acceptable pour certains tests de criblage de laboratoire pour la cocaïne, les opiacés et les cannabinoïdes $(15,24,39)$. Ces résultats ont été obtenus par comparaison des résultats issus des analyses $\mathrm{CG} / \mathrm{SM}$ ou $\mathrm{CG} / \mathrm{SM} / \mathrm{SM}$ en 
utilisant les valeurs seuil appropriées et des analyses immunochimiques des échantillons. Un test immunoenzymatique développé par Orasure Technologies a même reçu l'approbation de la FDA pour le criblage de la cocaïne, des cannabinoïdes et de la methamphétamine dans la salive. Les projets futurs incluent des tests de criblage de terrain pour les drogues dans la salive.

La confirmation des résultats des tests de criblage positifs nécessite un outil analytique très sensible et spécifique. La plupart des laboratoires utilisent actuellement la CG/SM ; cependant les faibles concentrations par comparaison aux urines (pour les cannabinoïdes, par exemple) ainsi que la faible masse/volume d'échantillon disponible plaident fortement pour l'utilisation de techniques plus sensibles telles que la GC/SM avec ionisation chimique négative ou encore en version tandem SM. Enfin, de plus en plus de laboratoires ont trouvé dans les systèmes CLHP/SM ou CLHP/SM/SM des outils analytiques dignes d'une place indispen- sable. Les tableaux IV et V présentent les valeurs seuil proposées par le SAMSHA pour les analyses de criblage et de confirmation de la salive et des cheveux sur les lieux de travail aux USA.

\section{Conclusion}

La salive et les cheveux semblent répondre aux exigences nécessaires pour être ajoutés aux matrices déjà employées pour le dépistage des usagers de drogue en milieu professionnel. Les méthodes adéquates de criblage et de confirmation existent et les analytes cibles,appropriés ont été identifiés. Des programmes de contrôle de qualité ont été développés et des valeurs seuil ont été proposées par des organisations scientifiques. Un avenir proche verra probablement la certification de laboratoires dans le monde entier ainsi que l'introduction de dispositions légales dans les pays concernés.

Tableau IV : Seuils proposés pour les laboratoires effectuant des analyses de cheveux dans le dépistage des stupéfiants en milieu professionnel.

\begin{tabular}{|l|l|c|c|}
\hline Classes de Drogues & \multicolumn{1}{c|}{$\begin{array}{c}\text { Analytes } \\
\text { (*analyte cible pour le criblage) }\end{array}$} & Criblage & Confirmation \\
\cline { 3 - 4 } & Amphétamines & $500^{(1)}$ & $300^{(2)}$ \\
& d-methamphétamine* & & 300 \\
\hline Cocaïne & d-amphétamine & 500 & $1000^{(3)}$ \\
& Cocaïne* & & 100 \\
\hline Opiacés & Benzoylecgonine & 200 & 200 \\
& morphine* & & 200 \\
& codéine & & $200^{(4)}$ \\
\hline Marijuana & 6-MAM & 1 & 0.05 \\
\hline
\end{tabular}

(1) le test doit présenter une réactivité croisée significative avec la MDMA, la MDA et la MDEA (50 à $150 \%$ de réactivité croisée)

(2) au minimum $50 \mathrm{pg} / \mathrm{mg}$ d'amphétamine doivent être présents pour rapporter un résultat positif pour la méthamphétamine

(3) positif si la cocaïne est $\geq 1000 \mathrm{pg} / \mathrm{mg}$ et la benzoylecgonine $\geq 100 \mathrm{pg} / \mathrm{mg}$ et le rapport $\mathrm{BE} /$ cocaïne $\geq 0.10$

(4) la présence de 6-acétylmorphine ne peut être rapportée sauf si un autre opiacé est présent en concentration supérieure à la valeur seuil

Tableau $V$ : Seuils proposés pour les laboratoires effectuant des analyses de salive dans le dépistage des stupéfiants en milieu professionnel.

\begin{tabular}{|c|c|c|c|}
\hline \multirow[t]{2}{*}{ Classes de Drogues } & \multirow{2}{*}{$\begin{array}{c}\text { Analytes } \\
\text { (*analyte cible pour le criblage) }\end{array}$} & \multicolumn{2}{|c|}{ Valeurs seuil $\mu \mathrm{g} /$} \\
\hline & & Criblage & Confirmation \\
\hline Amphétamines & $\begin{array}{l}\text { d-methamphétamine* } \\
\text { d-amphétamine }\end{array}$ & $160^{(1)(2)}$ & $\begin{array}{l}160^{(2)} \\
160^{(2)}\end{array}$ \\
\hline Cocaïne & Benzoylecgonine & 20 & 8 \\
\hline Opiacés & $\begin{array}{l}\text { morphine* } \\
\text { 6-MAM }\end{array}$ & 40 & $\begin{array}{c}40 \\
4 \\
\end{array}$ \\
\hline Marijuana & $\Delta$-9-tétrahydrocannabinol* & 4 & 2 \\
\hline
\end{tabular}

(1) le test doit présenter une réactivité croisée significative avec la MDMA, la MDA et la MDEA (50 à $150 \%$ de réactivité croisée)

(2) la valeur la plus récente proposée par Niedbala pour les amphétamines est de $50 \mu \mathrm{g} / \mathrm{l}$ 


\section{Références}

1. Verstraete A.G., Pierce A. Workplace drug testing in Europe. Forensic Sci. Int. $2001 ; 121: 2-6$.

2. Caplan Y.H., Goldberger B.A. Alternative specimens for workplace drug testing.J. Anal. Toxicol. $2001 ; 25$ : 396-99.

3. Substance Abuse and Mental Health Administration, Mandatory guidelines for Federal workplace drug testing programs, Fed. Regist. 1988 ; 53 : 11970-89.

4. de la Torre R., Segura J., de Zeeuw R., Williams J. Recommendations for the reliable detection of illicit drugs in urine in the European Union with special attention to the workplace. Ann. Clin. Biochem. 1997 ; 34 : 339-44.

5. UNDCP, Guidelines for testing under international control in hair, sweat and saliva, UN, New-York, 1998.

6. Substance Abuse and Mental Health Administration. Mandatory guidelines for federal workplace drug testing programs 2000. Available from http://www.health.org/ workplace/manguidelines/draft3.htm

7. Society of Hair Testing, Statement of the Society of Hair Testing concerning the examination of drugs in human hair. Forensic Sci. Int. 1997 ; 84 : 3-6.

8. Deveaux M., Kintz P., Goullé J.P., Bessard J., Pepin G., Gosset D. The hair analysis proficiency testing program of the French society of analytical toxicology. Forensic Sci. Int. 2000 ; 107 : 389-94.

9. Cone E. Legal, workplace, and treatment drug testing with alternate biological matrices on a global scale. Forensic Sci. Int. $2001 ; 121: 7-15$.

10. Samyn N., Verstraete A., van Haeren C., Kintz P. Analysis of drugs of abuse in saliva. Forensic Sci. Rev. 1999 ; 11 : 1-19.

11. Cone E.J. Saliva testing for drugs of abuse. Ann. N. Y. Acad. Sci. $1993 ; 694: 91-127$.

12. Höld K.M., de Boer D., Zuidema J., Maes R.A.A. Saliva as an analytical tool in toxicology. Int. J. Drug Testing $1996 ; 1$ : 1-36.

13. Haeckel R., Hänecke P. Application of saliva for drug monitoring: an in vivo model for transmembrane transport. Eur. J. Clin. Chem. Clin. Biochem. 1996 ; 34 : 171-91.

14. Samyn N., Viaene B., Laeremans B., De Boeck G. Evaluation of different roadside drug testing equipment. Contribution of Belgium. Deliverable D4 ROSITA Contract DG VII RO 98SC.3032, 2000 (available from http://www.rosita.org).

15. Niedbala R.S., Kardos K.W., Fritch D.F., Kardos S., Fries T., Waga I R sub J., Cone E. Detection of marijuana use by oral fluid anci urine analysis following single-dose administration of smoked and oral marijuana. J. Anal. Toxicol. 2001; 25 :289-303.

16. Huestis M.A., Henningfield J.E., Cone E.J. Blood cannabinoids. I. Absorption of THC and formation of 11-OH-THC and THC-COOH during and after smoking marijuana. J. Anal. Toxicol. $1992 ; 16: 276-82$.

17. Navarro M., Pichini S., Farré M., Ortuno J., Roset P.N., Segura J., de la Torre R. Usefulness of saliva for measurement of 3,4methylenedioxymethamphetamine and its metabolites: correlation with plasma drug concentrations and effect of salivary $\mathrm{pH}$. Clin. Chem. $2001 ; 47$ : 1788-95.

18. Samyn N., De Boeck G., Wood M., Lamers C.T.J., De Waard D., Brookhuis K.A., Verstraete A.G., Riedel W.J. Plasma, oral fluid and sweat wipe ecstasy concentrations in controlled and real-life conditions. Forensic Sci. Int. 2002, in press.

19. Cone E.J., Oyler J.M., Darwin W.D. Cocaine disposition in saliva following intravenous, intranasal, and smoked administration. J. Anal. Toxicol. $1997 ; 21:$ 465-75.
20. Moolchan E.T., Cone E.J., Wstadik A., Huestis M.A., Preston K.L. Cocaine and metabolite elimination patterns in chronic cocaine users during cessation : plasma and saliva analysis. J. Anal. Toxicol. $2000 ; 24: 458-66$.

21. Yacoubian G.S., Wish E.D., Perez D.M. A comparison of saliva testing to urinalysis in an arrestee population. J. Psychoactive Drugs $2001 ; 33$ : 289-94.

22. Jenkins A.J., Oyler J.M., Cone E.J. Comparison of heroin and cocaine concentrations in saliva with concentrations in blood and plasma. J. Anal. Toxicol. $1995 ; 19: 359-74$.

23. O'Neal C.L., Crouch D.J., Rollins D.E., Fatah A., Cheever M.L. Correlation of saliva codeine concentrations with plasma concentrations after oral codeine administration. J. Anal. Toxicol. $1999 ; 23: 452-59$.

24. Niedbala S., Kardos K., Waga J., Fritch D., Yeager L., Doddamane S., Schoener E. Laboratory analysis of remotely collected oral fluid specimens for opiates by immunoassay. J. Anal. Toxicol. $2001 ; 25: 310-15$.

25. O'Neal C.L., Crouch D.J., Rollins D.E., Fatah A.A. The effects of collection methods on oral fluid codeine concentrations. J. Anal. Toxicol. $2000 ; 24$ : 536-42.

26. Sachs H. Forensic applications of hair analysis. In : Kintz P., ed. Drug Testing in Hair. Boca Raton : CRC Press, 1996 ; 211-22.

27. Kintz P. Clinical applications of hair analysis. In : Kintz P., ed. Drug Testing in Hair. Boca Raton : CRC Press, 1996 ; 267-77.

28. Du Pont R.L., Baumgartner W.A. Drug testing by urine and hair analysis : complementary features and scientific issues. Forensic Sci. Int. 1995 ; 70 : 63-76.

29. Gaillard Y., Vayssette F., Pépin G. Compared interest between hair analysis and urinalysis in doping controls. Results for amphetamines, corticosteroids and anabolic steroids in racing cyclists. Forensic Sci. Int. $2000 ; 107$ : 361-79.

30. Kidwell D.A., Blank D.L. Environmental exposure - the stumbling block of hair testing. In : Kintz P., ed. Drug Testing in Hair. Boca Raton : CRC Press, 1996 ; 17-68.

31. Jurado C., Kintz P., Menendez M., Repetto M. Influence of the cosmetic treatment of hair on drug testing. Int. J. Legal Med. $1997 ; 110: 159-63$.

32. Cone E.J., Joseph R. The potential for bias in hair testing for drugs of abuse. In : Kintz P., ed. Drug Testing in Hair. Boca Raton : CRC Press, 1996 ; 69-93.

33. Baumgartner W.A., Hill V.A., Kippenberger D. Workplace drug testing by hair analysis : advantages and issues. In : Mieczkowski T., ed. Drug testing technology. Boca Raton : CRC Press, 1999 ; 283-310.

34. Rohrich J., Zorntlein S., Potsch L., Skopp G., Becker J. Effect of the shampoo Ultra Clean on drug concentrations in human hair. Int. J. Legal Med. 2000 ; 113 : 102-6.

35. Kintz P. Matrices alternatives et toxicologie médicolégale. In : Kintz P. (coordinateur), Toxicologie et pharmacologie médicolégale. Paris : Elsevier, 1998 ; 685-710.

36. Cone E.J., Yousefnejad D., Darwin W.D., Maguire T. Testing human hair for drugs of abuse. II. Identification of unique cocaine metabolites in hair of drug abusers and evaluation of decontamination procedures. J. Anal. Toxicol. $1991 ; 15: 250-55$.

37. Pépin G., Gaillard Y. Concordance between self-reported drug use and findings in hair about cocaine and heroin. Forensic Sci. Int. $1997 ; 84$ : 37-41.

38. Spiehler V. Hair analysis by immunological methods from the beginning to 2000. Forensic Sci. Int. $2000 ; 107$ : 249-59.

39. Niedbala R.S., Kardos K., Fries T., Cannon A., Davis A. Immunoassay for detection of cocaine/metabolites in oral fluids. J. Anal. Toxicol. $2001 ; 25: 62-8$. 